We are grateful to Dr W Fraser for vitamin D and PTHrP measurements and to Dr A White for ACTH and ACTH precursor measurements.

1 Paterson CR, Naismith KI, Young JA. Severe unexplained hypophosphatemia. Clin Chem 1992;38:104-7.

2 Drezner MK, Fenglos MN. Osteomalacia due to 125 dihydroxy-cholecalciferol deficiency: association with giant cell tumour of bone. $₹$ Clin Invest 1977;60: 1046-53.

3 Taylor HC, Fallon MD, Velasco ME. Oncogenic osteomalacia and inappropriate antidiuretic hormone secretion due to oat-cell carcinoma. Ann Intern Med 1984;101:786-8.
4 Ratcliffe JG. Endocrine effects of tumours. In: McGee JO'D, Isaacson, PG, Wright NA, eds. Oxford textbook of pathology. Vol 1. Oxford: OUP, 1992:694-703.

5 Ryan EA, Reiss E. Oncogenous osteomalacia: review of world literature of 42 cases and report of two new cases. Am ₹ Med 1984;77:501-12.

6 Aschinber LC, Solomon LM, Zeis PM, et al. Vitamin resistant rickets associated with epidermal nevus syndrome. $¥$ Pediatr 1977;91:56.

7 Seshadri MS, Cornish CJ, Mason RS, et al. Parathyroid hormone-like bioactivity in tumours from patients with ormone-like biomicin

8 Hewison M, Karmali R O'Riordan ILH. Tumou induced M, Karmali R, Oriordan JLH. Tumo

\title{
Gram negative septicaemia diagnosed on peripheral blood smear appearances
}

\author{
A Fife, D Hill, C Barton, P Burden
}

\begin{abstract}
Buffy coat smears from febrile patients may contain visible bacteria and therefore detect bacteraemia before conventional blood cultures become positive. However, it is unusual to see microorganisms in an otherwise untreated peripheral blood smear. The case of a febrile neutropenic patient is reported. A Wright's stained peripheral blood smear contained bacterial elements, thus making earlier diagnosis of septicaemia and identification of the causative bacterium possible.
\end{abstract}

$(\mathcal{F}$ Clin Pathol 1994;47:82-84)

\section{Case report}

A 59 year old woman was undergoing chemotherapy with ifosfamide and epirubicin for recurrent adenocarcinoma of the ovary following a disease free period of five years. Response to carboplatin had been limited and the patient had undergone surgical debulking of the residual tumour some five months earlier. A Hickman line had been inserted and the ifosfamide/epirubicin regimen started six weeks before the episode described here. The patient had already had a septicaemic episode (not associated with neutropenia) one month earlier. Two sets of blood cultures (cultured by the Sentinel method, Difco, UK) taken at this time were positive for Staphylococcus aureus and one set had also yielded Klebsiella oxytoca. She responded well to flucloxacillin and gentamicin.

She had undergone her third course of ifosfamide and epirubicin 10 days earlier and this had been complicated by the transient development of signs of ifosfamide toxicity which completely resolved.

A week after discharge she was admitted with a 24 hour history of rigors, vomiting, and epistaxis. Her general practitioner had given her oral cephradine the day before admission.

Examination showed that the patient was pale and obviously unwell. She was initially apyrexial. Despite the reported epistaxis there was no other evidence of abnormal bleeding. Her pulse rate was 84 per minute and her blood pressure 120/70 mm $\mathrm{Hg}$. Apart from mild ankle oedema there were no other cardiovascular signs and the respiratory system was also normal. The abdomen was soft and non-tender with no hepatosplenomegaly. She did not appear to be confused and there were no focal neurological signs.

A set of blood cultures was taken from the Hickman line, along with samples for full blood count, urea, and electrolytes and liver function tests. A mid-stream urine specimen was also obtained and the patient was given cefuroxime intravenously. Results of the initial investigations showed a haemoglobin concentration of $71 \mathrm{~g} / 1$ and a profound leucopenia of $0.2 \times 10^{9} / 1$. Her platelet count was also low at $31 \times 10^{9} / 1$. She was hypokalaemic with a potassium concentration of $2.1 \mathrm{mmol} / 1$ and had evidence of mild renal impairment which had not been present before.

Soon after admission the patient deteriorated and showed overt signs of sepsis. Her temperature rose to $39^{\circ} \mathrm{C}$ and she developed a tachycardia of 100 per minute which was accompanied by a drop in blood pressure to $100 / 60 \mathrm{~mm} \mathrm{Hg}$. A urinary catheter was inserted to monitor output and resuscitation with intravenous fluids begun. Her antibiotic treatment was changed to piperacillin $4 \mathrm{~g}$ four times daily and gentamicin $80 \mathrm{mg}$ three times daily in keeping with the regimen for febrile neutropenia in use in this hospital. She was given blood and platelet support.
Correspondence to: Dr A J Fife

Accepted for publication 5 July 1993 


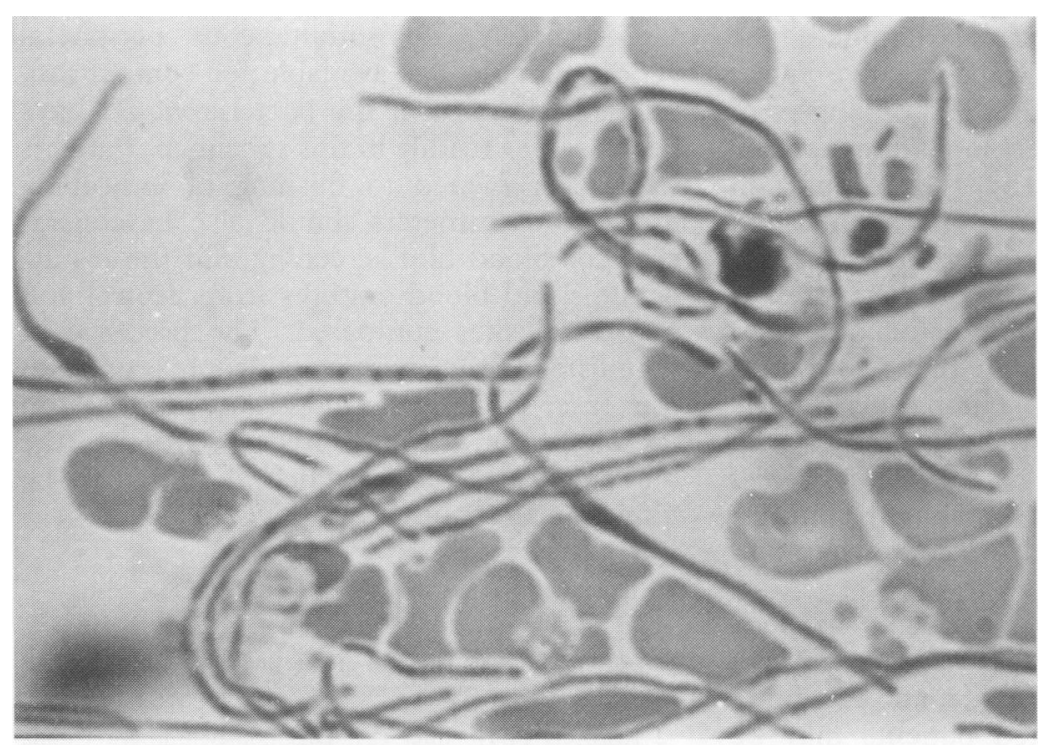

Wright's stained peripheral blood smear.

\section{Discussion}

The use of Wright's stained buffy coat smears in the early detection of bacteraemia was first described in $1944 .{ }^{1}$ However, the method has never been popular as a diagnostic tool because of its lack of sensitivity in detecting bacteraemia when compared with conventional blood culture methods. Later attempts to improve the yield of positive results using Gram and Giemsa stained preparations also gave disappointing results and the method fell into disuse. A more recent study compared acridine orange and Gram stained buffy coat smears with the results of blood culture in patients thought clinically to be bacteraemic. ${ }^{2}$ Seventy six per cent of patients with positive blood cultures had demonstrable bacteria in acridine orange stained buffy coat preparations. This figure dropped to $52 \%$ when Gram stained smears were examined. Despite the improved sensitivity of the acridine orange method, its significant false negative rate and the laboratory time and labour which would be required to screen blood smears from all patients in whom blood culture is indicated, make it unlikely to become widely used. Most patients thought to be clinically septicaemic are in any case treated empirically with broad spectrum antibiotics pending the results of an infective screen including blood culture.

Despite the fact that buffy coat examination is a relatively insensitive test when applied prospectively to large numbers of patients, there are occasions when the peripheral blood smears of bacteraemic patients are found to contain micro-organisms.

$S$ mith $^{3}$ found that infected leucocytes were visible in smears prepared from earlobe capillary blood of patients with suspected septicaemia and suggested that this may form the basis of a screening test. This method was insensitive when compared with blood culture results and has not been widely used. However, the fortuitous finding of microorganisms in the peripheral blood smear can, as in the case of our patient, lead to earlier diagnosis. Clostridium perfringens septicaemia, post splenectomy pneumococcal sepsis, and staphylococcal infections related to long term intravenous catheters have also been diagnosed on the peripheral blood film appearances (Barton $\mathrm{C}$, personal communication). In another case report cocci were seen within leucocytes on a conventionally prepared peripheral blood film. Subsequent Gram staining of the peripheral blood showed them to be Gram negative diplococci. This was the first clue to the diagnosis in a woman who died of circulatory collapse and disseminated intravascular coagulation within hours of admission to hospital. Neisseria meningitidis was isolated from blood cultures taken at admission and from post mortem cerebrospinal fluid.

Although detection of micro-organisms in peripheral blood is undoubtedly a useful finding, care should be taken in interpreting the morphological appearances in the film, as illustrated by our case. When films from the episode during which coagulase negativ staphylococci were isolated from the blood cultures. Culture of the line tip showed the presence of a coliform which was identified as Klebsiella species. 
blood culture bottles were examined, the organisms appeared as Gram negative bacilli typical of the enterobacteria. The dangers of overinterpretation of the blood film appearances are demonstrated by a case report in which bacteria seen in a Wright's stained film were thought to have the morphology of Neisseria sp. ${ }^{5}$ The patient was a very premature infant and was given cefotaxime and ampicillin on the basis of the blood film finding. Subsequent Gram staining of a buffy coat preparation showed the bacteria to be Gram positive cocci and Staphylococcus epidermidis was isolated from blood cultures. Antibiotics were changed to vancomycin and the patient recovered.

The finding of micro-organisms in peripheral blood smears should be regarded as a useful indicator of bacteraemia, and screening of blood films in selected patients may be worth while. Patients with febrile neutropenia and those who have had their spleen removed and admitted with fever should be considered. The fact that our patient's bacteraemia was almost certainly due to a colonised intravenous line would explain the ease with which micro-organisms were seen in the film prepared from blood taken from the catheter.
Unfortunately, no simultaneous peripheral venous sample was available for comparison, but it is doubtful if the bacteria would have been seen as readily in this specimen. Patients with fever related to flushing of indwelling intravenous catheters should also be considered for blood film screening and the results of films and blood cultures from central and peripheral sites compared. The presence of organisms, either within neutrophils or as in this case, in clinically important numbers on the blood film, should direct attention to the blood culture bottles which should then be filmed and subcultured.

We thank Dr J Kremer for permission to report this case.

1 Humphrey AA. The use of the buffy layer in the rapid diagnosis of septicaemia. Am $\mathcal{F}$ Clin Pathol 1944;14: 358-62

2 Ristuccia P, Hoeffner RA, Digamon-Beltran M, Cunha BA. Detection of bacteraemia by buffy coat smears. Scand $\mathcal{F}$ Infect Dis 1987;19:215-7.

3 Smith $\mathbf{H}$. Leucocytes containing bacteria in plain blood films from patients with septicaemia. Aust Ann Med 1962;15:210-21.

4 Scott MA, Gobert-Jones J. Intracellular organisms in peripheral blood in a case of meningococcal septiperipheral blood in a case of meningoco

5 Selby DM, Gautier G, Luban NLC, Campos JM. Overwhelming neonatal septicaemia diagnosed upon examination of peripheral blood smears. Clin Pediatr 1990;29:706-9. 